\title{
Basic features of proton-proton interactions at ultra-relativistic energies and RFT-based quark-gluon string model
}

\author{
E. Zabrodin ${ }^{1,2,3, a}$, L. Bravina ${ }^{2,3}$, and J. Bleibel ${ }^{4,5}$ \\ ${ }^{1}$ Skobeltsyn Institute of Nuclear Physics, Moscow State University, RU-119991 Moscow, Russia \\ ${ }^{2}$ Department of Physics, University of Oslo, PB 1048 Blindern, N-0316 Oslo, Norway \\ ${ }^{3}$ National Research Nuclear University "MEPhl" (Moscow Engineering Physics Institute), Kashirskoe high- \\ way 31, Moscow, RU-115409, Russia \\ ${ }^{4}$ Max-Planck-Institut für Intelligente Systeme, Heisenbergstr. 3, D-70569 Stuttgart, Germany \\ ${ }^{5}$ Institut für Augewandte Physik, Universität Tübingen, Auf der Morgenstelle 10, D-72076 Tübingen, Ger- \\ many
}

\begin{abstract}
Proton-proton collisions at energies from $\sqrt{s}=200 \mathrm{GeV}$ up to $\sqrt{s}=14 \mathrm{TeV}$ are studied within the microscopic quark-gluon string model. The model is based on Gribov's Reggeon Field Theory accomplished by string phenomenology. Comparison with experimental data shows that QGSM describes well particle yields, rapidity- and transverse momentum spectra, rise of mean $\left\langle p_{T}\right\rangle$ and forward-backward multiplicity correlations. The latter arise in QGSM because of the addition of various processes with different mean multiplicities. The model also indicates fulfillment of extended longitudinal scaling and violation of Koba-Nielsen-Olesen scaling at LHC. The origin of both features is traced to short-range particle correlations in the strings. Predictions are made for $\sqrt{s}=14 \mathrm{TeV}$.
\end{abstract}

\section{Introduction}

Despite the significant progress achieved in the last years, the theory of multiparticle production in elementary proton-proton collisions at ultrarelativistic energies is not completed yet. We all know, of course, that the processes with large momentum transfer $Q^{2}$ are well described by the perturbative chromodynamics (pQCD). Unfortunately, even at very high energies the main contribution to multiparticle production in hadronic interactions comes from the processes with small momentum transfer. This means that the running coupling constant $\alpha_{s}\left(Q^{2}\right)$ is not small and, therefore, the perturbative series expansion is not very helpful. Other techniques, based on non-perturbative methods, should be utilized. The quark-gluon string model (QGSM) [1] and similar to it dual parton model (DPM) [2] is one of the possible approaches to solution of this very interesting and difficult problem. Both models are based on the Reggeon Field theory (RFT) [3]. Basic principles of the QGSM are sketched below.

\footnotetext{
a e-mail: eugen.zabrodin@fys.uio.no
} 


\section{The QGSM model}

The quark-gluon string model, formulated about 35 years ago [1], employs the $1 / N$ series expansion of the amplitude of a process in QCD, where $N$ is either the number of colors [4] or the number of flavors [5]. This method is also called topological expansion, because of emergence of diagrams of various topologies. Although it is not possible to assign weights for the diagrams within the QCD, there is one-to-one mapping between the diagrams in $1 / N$-expansion and the processes with exchange of Regge singularities in the $t$-channel. For instance, exchange of quantum numbers via Reggeons corresponds to planar diagrams, whereas the cylinder diagrams are represented by the reactions without the quantum number exchange. The latter proceed via the exchange of Pomerons. Therefore, the perturbative Reggeon Field Theory (RFT) [3] is directly linked to quantum chromodynamics.

The Monte Carlo version of the QGSM [6] employs statistical weights, hadron structure functions and leading quark fragmentation functions obtained from the Regge approach in [1] to choose subprocesses of string production, to compute mass and momentum of strings and to simulate string decays, respectively. The hadron-hadron collision part of the model includes single and double diffraction subprocesses, antibaryon-baryon annihilation and elastic scattering. The hadron inelastic interaction cross section $\sigma_{\text {inel }}(s)=\sigma_{\text {tot }}(s)-\sigma_{e l}(s)$ is split further into the cross section for single diffractive interactions $\sigma_{S D}(s)$ and the cross section for non-diffractive reactions $\sigma_{N D}(s)$, similar to analysis of experimental data. By means of the Abramovskii-Gribov-Kancheli(AGK) cutting rules [7] the inelastic non-diffractive interaction cross section $\sigma_{N D}(s)$ can be expressed via the sum of the cross sections for the production of $n=1,2, \ldots$ pairs of quark-gluon strings, or cut Pomerons, and the cross section of double diffractive process

$$
\sigma_{N D}(s)=\sum_{n=1}^{\infty} \sigma_{n}(s)+\sigma_{D D}(s) .
$$

To find $\sigma_{n}(s)$ one can utilize the quasi-eikonal model $[8,9]$ which states that

$$
\begin{aligned}
\sigma_{\text {tot }}(s) & =\sum_{n=0}^{\infty} \sigma_{n}(s)=\sigma_{P} f\left(\frac{z}{2}\right), \\
\sigma_{n}(s) & =\frac{\sigma_{P}}{n z}\left(1-\exp (-z) \sum_{k=0}^{n-1} \frac{z^{k}}{k !}\right), k \geq 1 \\
\sigma_{0} & =\sigma_{P}\left(f\left(\frac{z}{2}\right)-f(z)\right), \\
f(z) & =\sum_{v=1}^{\infty} \frac{(-z)^{v-1}}{v v !},
\end{aligned}
$$

Here

$$
\begin{aligned}
\sigma_{P} & =8 \pi \gamma_{P} \exp (\Delta \xi) \\
z & =\frac{2 C \gamma_{P}}{\left(R_{P}^{2}+\alpha_{P}^{\prime} \xi\right)} \exp (\Delta \xi)
\end{aligned}
$$

The cross section $\sigma_{0}$ corresponds to diffraction contribution. The parameters $\gamma_{P}$ and $R_{P}$ are Pomeronnucleon vertex parameters, quantity $\Delta \equiv \alpha_{P}(0)-1$, and $\alpha_{P}(0)$ and $\alpha_{P}^{\prime}$ is the intercept and the slope of the Pomeron trajectory, respectively. The quantity $C$ takes into account the deviation from the pure eikonal approximation $(C=1)$ due to intermediate inelastic diffractive states, $\xi=\ln \left(s / s_{0}\right)$ and $s_{0}$ is a scale parameter. Note that at very high energies the enhanced diagrams [10], describing the multiple 
Table 1. Parameters of both soft and hard Pomeron used in the present calculations.

\begin{tabular}{ccc}
\hline \hline Parameter & Soft Pomeron & Hard Pomeron \\
\hline \hline$\alpha_{P}(0)$ & 1.15615 & 1.3217 \\
$\alpha_{P}^{\prime}$ & 0.25 & 0 \\
$R_{P}$ & 2.0 & 2.4 \\
$\gamma_{P}$ & 1.27475 & 0.021 \\
\hline
\end{tabular}

gluon production from Pomeron vertices, can violate [11] the AGK cutting rules. These diagrams, however, are not implemented in the MC version of QGSM employed for present calculations.

At ultrarelativistic energies the model includes the new processes which represent the hard (multi)Pomeron exchanges. - The concept of soft and (semi)hard Pomerons is attributed to a major part of Monte Carlo models based on RFT, see [2, 12-14]. - Parameters of the soft and hard Pomerons used in present calculations are listed in Table 2. We see that the intercept of hard Pomeron trajectory is significantly larger than that of the soft Pomeron, whereas its slope is zero.

The diagrams describing subprocesses in ultrarelativistic $p p$ collisions are displayed in Fig. 1. Here diagram (a) shows the soft multi-Pomeron exchange, diagram (b) represents both hard and soft Pomeron exchange, diagrams (c) and (d) show the single diffractive (SD) process with small (c) and large (d) mass excitation, and diagrams (e)-(g) display double diffractive (DD) processes with large (e) and small (f) mass excitation, and central diffraction (g). The cross sections of SD and DD processes in $p p$ collisions were calculated within the QGSM in [15]. In our MC version, we utilize the following parametrization of these results proposed in [16]

$$
\begin{aligned}
& \sigma_{S D}(s)=0.68\left(1+\frac{36}{s}\right) \ln (0.2 s)+0.6 \\
& \sigma_{D D}(s)=0.27 \ln s+1.65
\end{aligned}
$$

Cross sections of both processes rise as $\sigma_{D} \propto \ln s$, which satisfies the Froissart limit $\sigma_{t o t} \propto(\ln s)^{2}$.

Cross sections $\sigma_{t o t}, \sigma_{e l}, \sigma_{S D}$ and $\sigma_{D D}$ obtained by QGSM are compared in Fig. 2 with the predictions of other RFT-based models [14, 17-20]. At Tevatron energy $\sqrt{s}=1.8 \mathrm{TeV}$ all models agree within 5\% accuracy limit for all cross sections except of the double-diffraction one, whereas at $\sqrt{s}=14 \mathrm{TeV}$ the model predictions start to deviate. Still, results for $\sigma_{\text {tot }}$ and $\sigma_{S D}$ are close to each other. Two versions of the Durham model, KMR-1 and KMR-2, predict excess of about $50 \%$ of double-diffraction cross section compared to other models. QGSM results are quite close to the calculations of the GLMM model [17]. QGSJET model [14], which also employs the scheme with soft and hard Pomerons, yields larger total and elastic cross sections at $\sqrt{s}=14 \mathrm{TeV}$. Further discussion of the differences and similarities between the models can be found in [18].

It is easy to see that the main contribution to particle production in $p p$ collisions at ultrarelativistic energies comes from the multi-Pomeron exchanges. The amplitude of the $n$-Pomeron exchange is suppressed by factor $\left(\frac{1}{N}\right)^{2 n}$, but it grows proportionally to $\left(\frac{s}{s_{0}}\right)^{n \Delta}$. The last term dominates over the suppression factor at LHC energies.

Finally, the Field-Feynman algorithm [21] is employed to describe the fragmentation of strings into hadrons. Here the hadron emission proceeds from both ends of the string with equal probabilities under imposed conditions of the preservation of the quark numbers and the energy-momentum conser- 

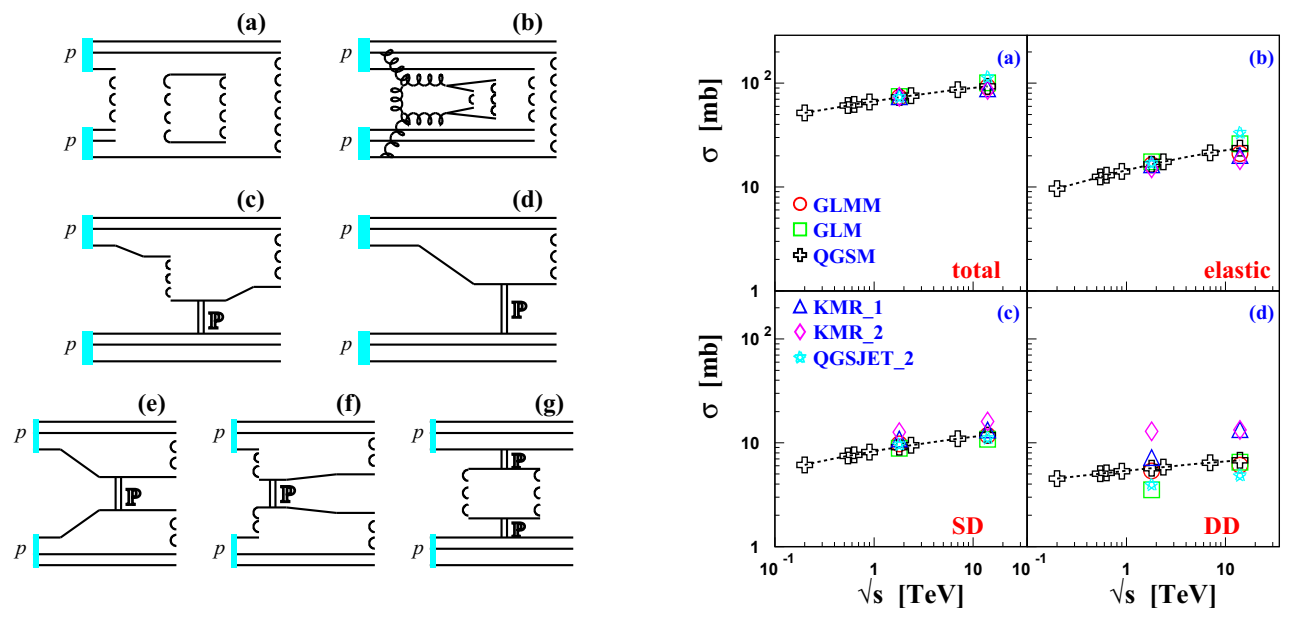

Figure 1. Diagrams taken into account in QGSM in Figure 2. Model predictions for (a) total, (b) elastic, (c) the modeling of $p p$ interactions at ultrarelativistic en- single-diffractive and (d) double-diffractive cross secergies: (a) multi-Pomeron exchange, (b) (semi)hard tions as functions of $\sqrt{s}$. The models are as follows: gluon-gluon interaction and soft Pomeron exchange, GLMM (circles) [17], GLM (squares) [18], QGSM (c)-(d) single diffraction with high-mass and low-mass (crosses), KMR-1 (triangles) [19], KMR-2 (diamonds) excitation, (e)-(f) double diffraction with low-mass and [20], and QGSJET-2 (stars) [14]. Dashed lines between high-mass excitation, (g) central diffraction. the QGSM points are drawn to guide the eye.

vation. The transverse momenta of the produced quarks and diquarks obey the power-law probability

$$
\begin{aligned}
f\left(p_{T}^{2}\right) d p_{T}^{2} & =\frac{3 D b_{2}(s)}{\pi}\left(1+D p_{T}^{2}\right)^{-4} d p_{T}^{2}, \\
b_{2}(s) & =0.325+0.016 \ln s, \quad D=0.34(\mathrm{GeV} / c)^{-2}
\end{aligned}
$$

QGSM was successfully extended to the description of hadron-nucleus and nucleus-nucleus collisions at energies spanning from several GeV up to energies available at RHIC and LHC, see, e.g., [22-30]. Our present study, however, deals merely with proton-proton collisions in the energy range $200 \mathrm{GeV} \leq \sqrt{s} \leq 14 \mathrm{TeV}$.

\section{Results}

\subsection{Transverse momentum and rapidity distributions}

Here results for inelastic and non-single diffractive $p p$ collisions are presented. Figure 3 displays dependence of the invariant cross section $E \frac{d^{3} \sigma}{d p^{3}}$ of charged hadrons on their transverse momentum $p_{T}$ in NSD events. Calculations are performed for c.m. energies varying from $200 \mathrm{GeV}$ to $14 \mathrm{TeV}$. Experimental data used for the comparison are taken from [31-43]. One can see that the combination of soft and hard processes in the model describes fairly well the $p_{T}$-spectra in the interval $0 \leq p_{T} \leq$ $10 \mathrm{GeV} / c$ for all energies. The slopes of the distributions become less steep with rising collision 

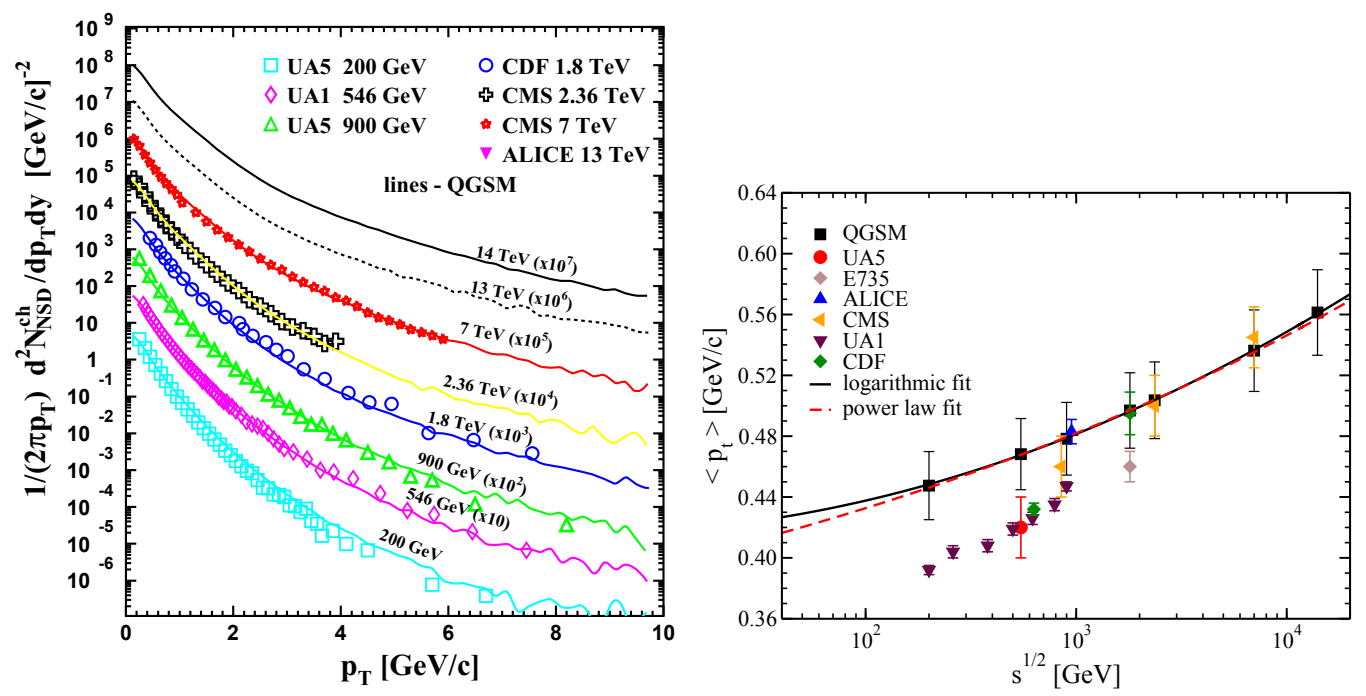

Figure 3. Transverse momentum distributions of the Figure 4. $\left\langle p_{T}\right\rangle$ vs. $\sqrt{s}$. Squares indicate the QGSM invariant cross section of charged particles in NSD $p p$ results; other symbols show the experimental data from collisions obtained in QGSM at $|y| \leq 2.5$ for energies [31-34, 37, 41]. Solid and dashed lines present fit to from $200 \mathrm{GeV}$ up to $14 \mathrm{TeV}$. Data are taken from [31- Eq. (12) and Eq. (13), respectively.

$33,39,41]$.

energy, i.e. the spectra become harder because of the increasing contribution of the hard processes. This leads to increase of the average transverse momentum of charged hadrons shown in Fig. 4. Here the model results are plotted onto the experimental data, obtained in $p p$ and $\bar{p} p$ interactions at $\sqrt{s} \leq 7 \mathrm{TeV}$. The LHC data show clearly a faster rise of the mean $p_{T}$ compared to linear extrapolation $\left\langle p_{T}\right\rangle=a+b \ln s$ of data below $900 \mathrm{GeV}$. Two parametrizations of $\left\langle p_{T}\right\rangle$ were proposed. The first one is purely phenomenological quadratic dependence on $\ln s$, and the second one is a power-law dependence on $E=\sqrt{s} / 2$ obtained within the color glass condensate (CGC) approach [44, 45]. By fitting the QGSM results, we got the following parameters for both approximations

$$
\begin{aligned}
& \left\langle p_{\mathrm{T}}\right\rangle=0.417-0.0035 \ln s+0.00059 \ln ^{2} s, \\
& \left\langle p_{\mathrm{T}}\right\rangle=0.243+0.12 \mathrm{E}^{0.1107} .
\end{aligned}
$$

Both curves are shown in Fig. 4 also. We see that the curves almost coincide within the whole investigated energy range, and so it is impossible to discriminate between the two parametrizations. Note, however, that Eq. (13) contains one less parameter compared to Eq. (12) because the exponent 0.1107 is not a free parameter but equals to one half of the exponent of the power-law fit to c.m. energy dependence of magnitudes of $d N^{\text {charge }} / d \eta$ distributions at midrapidity [44, 45].

To check this correspondence, the $d N / d \eta$ distributions of charged hadrons in inelastic and NSD events are displayed in Fig. 5(a) and Fig. 5(b), respectively. Comparison with experimental data shows that QGSM correctly reproduces the data in both central and fragmentation region. The charged particle density at midrapidity increases with rising $\sqrt{s}$, i.e., no Feynman scaling [46] is observed at LHC. The model indicates further rise of the central charged particle density at $\sqrt{s}=14 \mathrm{TeV}$ of about $20 \%$ compared to that at $\sqrt{s}=7 \mathrm{TeV}$; the values at midrapidity are $d N^{\text {inel }} / d \eta=5.8$ and $d N^{N S D} / d \eta=$ 6.7 , respectively. It is interesting to compare these results with the predictions in Ref. [47] made by 

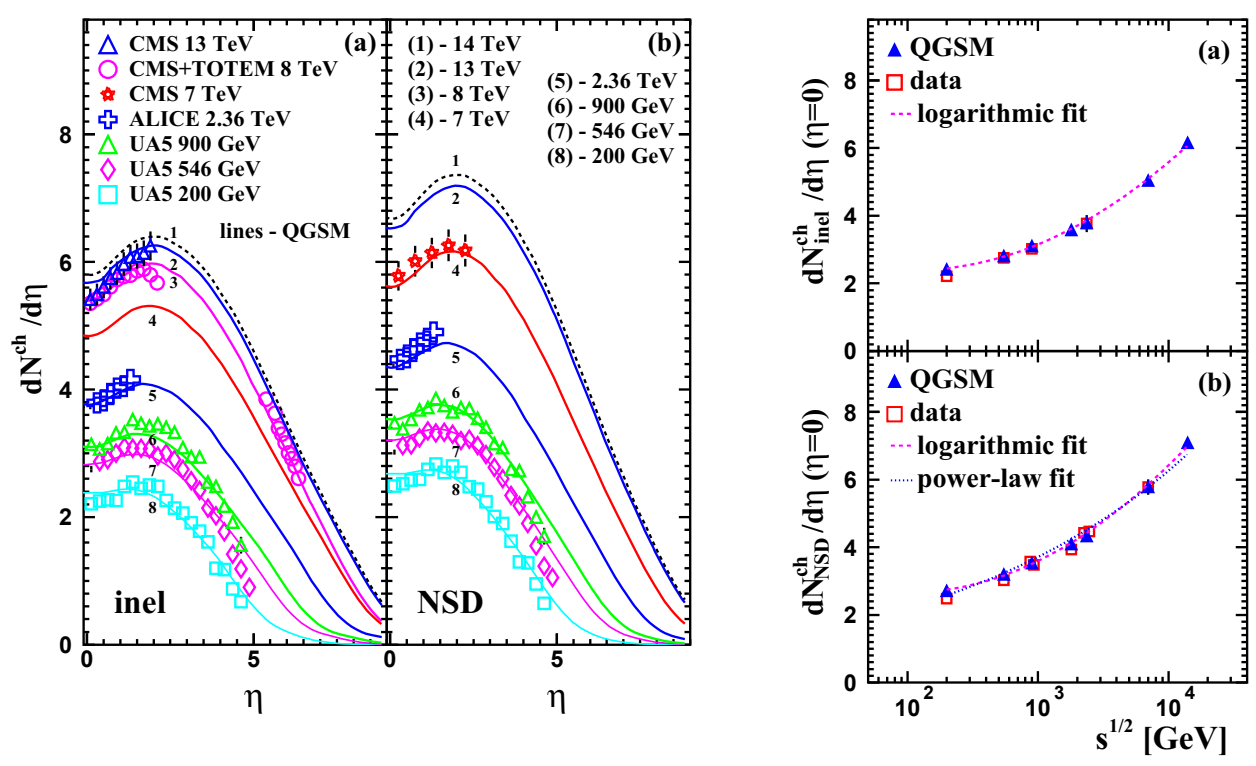

Figure 5. Pseudorapidity spectra of charged hadrons Figure 6. The density of charged particles at midrapidin (a) inelastic and (b) NSD $p p$ collisions at energies ity as a function of $\sqrt{s}$ for (a) inelastic and (b) NSD $p p$ from $200 \mathrm{GeV}$ up to $14 \mathrm{TeV}$. Lines show the QGSM collisions. Dashed lines show the results of the fit to calculations, symbols denote the data taken from [31, quadratic dependence on $\ln s$, whereas dotted line (b) $33,36,39,41,43]$. to the power-law dependence $\sim \sqrt{s}^{\lambda}$.

extrapolation of the data at lower energies. The extrapolation method relies on the proportionality to $\ln s$ of both the height and the width of $d N / d \eta$-distributions, and the energy independence of the slopes of $\eta$-spectra. This provides $d N^{\text {inel }} / d \eta(\eta=0)=4.6 \pm 0.4$ and $d N^{N S D} / d \eta(\eta=0)=5.25 \pm 0.7$ [47]. Both results are, obviously, significantly below the LHC experimental data.

The increase of midrapidity density as function of $\sqrt{s}$ is presented in Fig. 6 for both inelastic and non-single diffractive interactions. One can see clearly the deviation from the linear rise, proportional to $\ln s$, which is valid for energies below $2 \mathrm{TeV}$. Similarly to $p_{T}$ spectra, $d N / d \eta$ distributions calculated by QGSM were also fitted to the quadratic logarithmic dependence and to the power-law dependence, respectively, in the energy interval $200 \mathrm{GeV} \leq \sqrt{s} \leq 14 \mathrm{TeV}$. The results are as follows:

$$
\begin{aligned}
& \left.\frac{d N_{\text {inel }}}{d \eta}\right|_{\eta=0}(s)=4.36-0.507 \ln s+0.03 \ln ^{2} s, \\
& \left.\frac{d N_{N S D}}{d \eta}\right|_{\eta=0}(s)=5.015-0.60 \ln s+0.036 \ln ^{2} s \\
& \left.\frac{d N_{N S D}}{d \eta}\right|_{\eta=0}(s)=0.77 E^{0.22} .
\end{aligned}
$$

Both fitting curves are plotted onto the spectra in Fig. 6. Again, there is no visible difference between the two parametrizations. Note that the exponent $\lambda=0.22$ is indeed twice larger than the corresponding exponent of the power-law fit to the mean- $p_{T}$ data. 

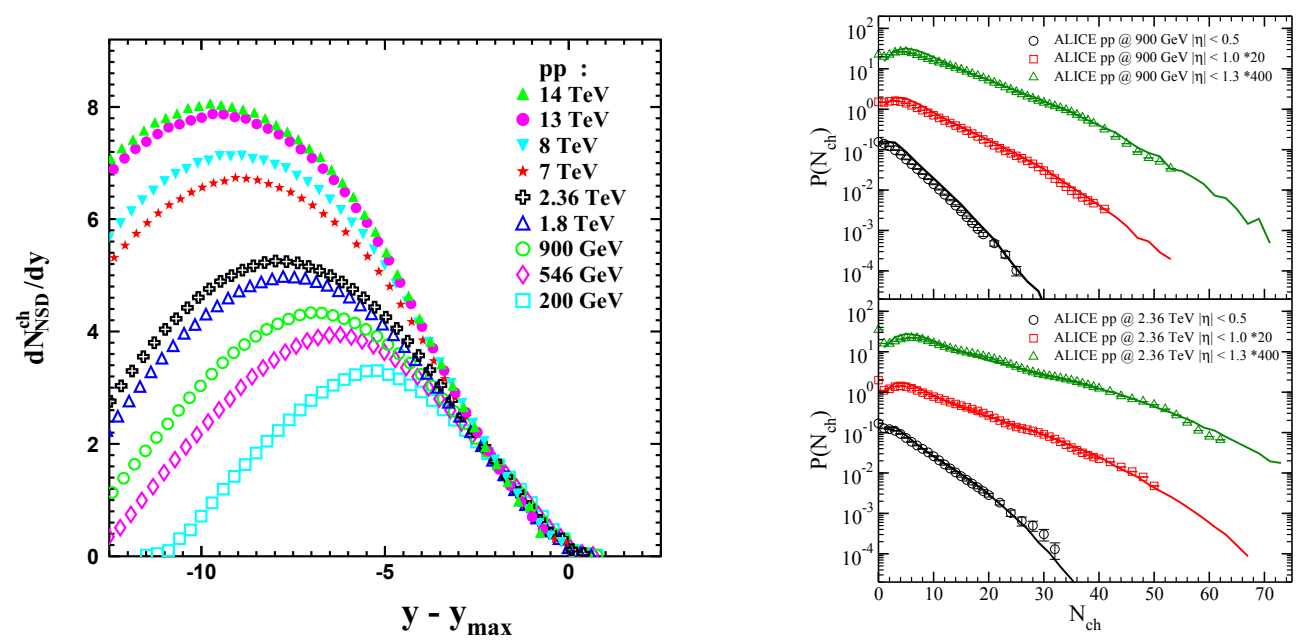

Figure 7. The distributions $\frac{1}{\sigma_{N S D}} \frac{d \sigma_{N S D}}{d y}$ of charged Figure 8. Charged particle multiplicity distributions in hadrons as functions of rapidity difference $y-y_{\max }$ obtained in QGSM for $p p$ collisions at all energies in question. $|\eta|<0.5,|\eta|<1.0$ and $|\eta|<1.3$ intervals, obtained in QGSM for $p p$ collisions at (a) $\sqrt{s}=900 \mathrm{GeV}$ and at (b) $\sqrt{s}=2.36 \mathrm{TeV}$. Open symbols denote the ALICE data [36].

\subsection{Extended longitudinal scaling and KNO scaling}

Extended longitudinal scaling (ELS) was observed first in Ref. [48] in the fragmentation region of $d N / d y$ (or $d N / d \eta$ ) spectra. Here the particle rapidity spectra have the same slope independent on the c.m. energy $\sqrt{s}$. To demonstrate this distinctly, the $d N / d y$ distributions at different energies are shifted to the beam rapidity, $y_{\max }$. The ELS was found to hold to $10 \%$ in a broad energy range $30.8 \mathrm{GeV} \leq \sqrt{s} \leq 900 \mathrm{GeV}$ [31]. Some time ago, in Ref. [49] the violation of the ELS in $p p$ collisions was predicted at energies available at LHC. To obtain the $d N / d y$ particle spectrum at LHC the authors of [49] have fitted the rapidity spectra of hadrons at lower energies to the Gaussian, extracted the widths $\sigma_{G}$, and extrapolated the obtained $\sigma_{G}(\sqrt{s})$ dependence to the LHC energy range. The predicted $d N / d y$ spectrum appeared to be quite narrow, thus indicating violation of the ELS.

QGSM does not support this prediction. We plot in Fig. $7 d N_{N S D}^{c h} / d y$ spectra as functions of $y-y_{\max }$ in $p p$ interactions at energies from $\sqrt{s}=200 \mathrm{GeV}$ to $14 \mathrm{TeV}$. It is easy to see that in the fragmentation region all curves sit on the top of each other. The fulfillment of the extended longitudinal scaling in the model is due to short-range rapidity correlations of hadrons in a string [16]. The correlation function of any two particles has exponential dependence

$$
C\left(y_{1}, y_{2}\right) \propto \exp \left[-\lambda\left(y_{1}-y_{2}\right)\right]
$$

therefore, particles are uncorrelated provided $\Delta y=y_{1}-y_{2} \gg 1$. Then, the $i$-th particle inclusive cross section for the process $1+2 \longrightarrow i+X$ is

$$
f_{i}=\frac{d^{2} \sigma\left(y_{1}-y_{i}, y_{i}-y_{2},\left(p_{T}^{(i)}\right)^{2}\right)}{d y_{i} d^{2}\left(p_{T}^{(i)}\right)^{2}}
$$




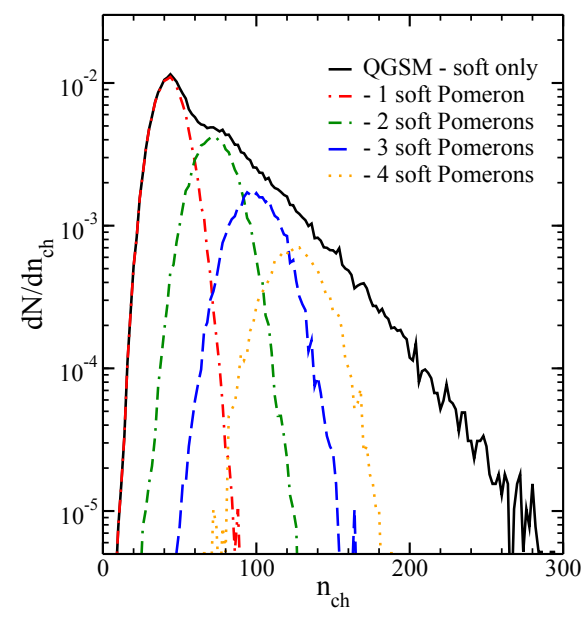

Figure 9. QGSM calculations of charged particle multiplicity distribution for processes going via the exchange of soft Pomerons in $p p$ collisions at $\sqrt{s}=14 \mathrm{TeV}$. Partial contributions of the 1P, 2P, 3P, and 4P processes are shown by dash-dotted, double-dash-dotted, dashed, and dotted lines, respectively.

If particle $i$ is produced in the fragmentation region of particle 1 , this means that $y_{1}-y_{i} \approx 1$ and $y_{i}-y_{2} \approx y_{1}-y_{2} \gg 1$, i.e., $f_{i}$ depends only on two variables, $y_{1}-y_{i}$ and $\left(p_{T}^{(i)}\right)^{2}$. Recalling that $\ln x_{F}^{(i)} \propto y_{i}-y_{1}$, the condition of the Feynman scaling for the particle inclusive density $n_{i}=f_{i} / \sigma_{\text {inel }}$ is recovered:

$$
n_{i}=\phi\left(x_{F}^{(i)},\left(p_{T}^{(i)}\right)^{2}\right) .
$$

Another interesting form of the scaling dependence is the so-called Koba-Nielsen-Olesen (KNO) scaling suggested in Ref. [50]. According to it, the multiplicity distribution $P_{n}(s)=\sigma_{n}(s) / \sigma_{t o t}(s)$, where $\sigma_{n}(s)$ is the partial cross section of the process with $n$ particles in the final state, depends on the c.m. energy $\sqrt{s}$ only through the normalized multiplicity $n /\langle n\rangle$, i.e.,

$$
P_{n}(s)=\frac{1}{\langle n\rangle} \Psi\left(\frac{n}{\langle n\rangle}\right) .
$$

Here $\langle n\rangle$ is the averaged multiplicity and $\Psi(n /\langle n\rangle)$ is an energy independent function. The KNO scaling was observed in $p p$ and $\bar{p} p$ collisions up to energies available at ISR accelerator, $\sqrt{s} \leq$ $62 \mathrm{GeV}$. Its violation at higher energies was predicted by the RFT specialists, see, e.g., [1, 7, 51]. The mechanism of the KNO violation within the Reggeon Field Theory is as follows.

The multiplicity of the produced particles in ultrarelativistic hadronic interactions is determined mainly by the number of cut Pomerons. Each cut Pomeron produces two strings, and the short range correlations of particles within the strings provide a Poisson multiplicity distribution of secondaries. At not very high energies or in the central rapidity window $|y| \leq 1$, the contributions of the processes with different number of cut Pomerons strongly overlap. Therefore, the KNO scaling approximately holds. With rising c.m. energy, the number of strings and the energy deposited into a single string increases. As a result, we have an enhancement of the high multiplicity events. The particle distribution starts to deviate from the Poissonian, and violation of the KNO scaling takes place.

The QGSM calculations of the charged particle multiplicity distributions in three midrapidity intervals for $p p$ interactions at $900 \mathrm{GeV}$ and $2.76 \mathrm{TeV}$ are compared in Fig. 8 with the experimental data 

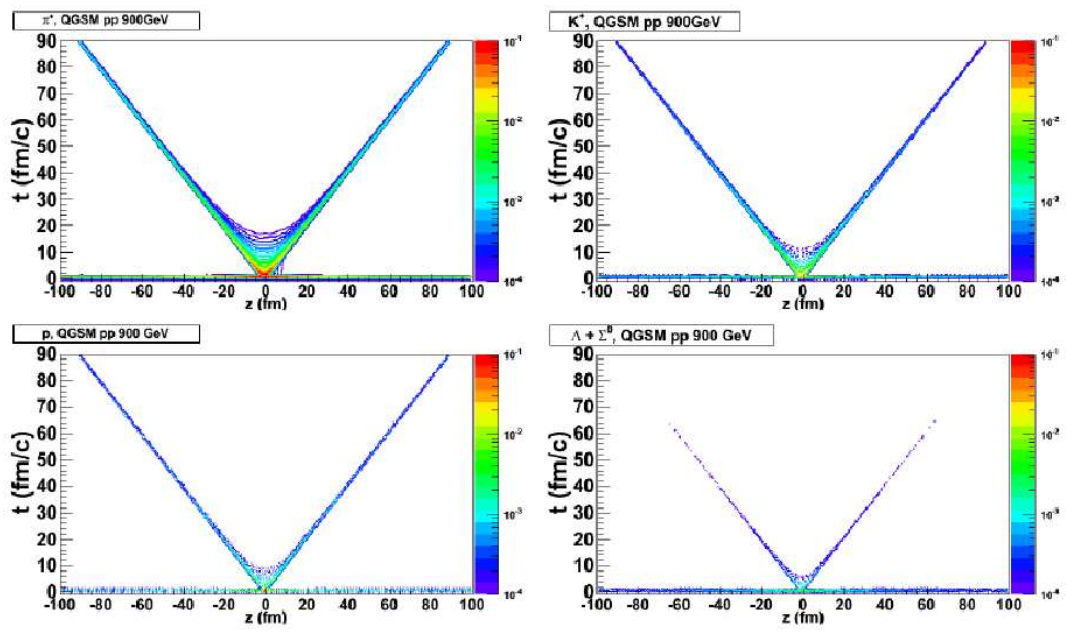

Figure 10. $d^{2} N / d z d t$ distribution of positive pions (upper left), positive kaons (upper right), protons (bottom left) and $\Lambda+\Sigma^{0}$ (bottom right) in QGSM for $p p$ collisions at $\sqrt{s}=900 \mathrm{GeV}$.

of the ALICE Collaboration [36]. The model results agree well with the data. Investigation of these spectra in terms of the variable $n /\langle n\rangle$, see Ref. [52], has confirmed the violation of the KNO scaling in $p p$ collisions at LHC energies. Namely, (i) formation of the more pronounced peak at not very high multiplicities, and (ii) rise of the high multiplicity tail of the multiplicity distribution have been observed. Figure 9 reveals the underlying mechanisms of these phenomena. For the sake of simplicity, we consider here events with only soft Pomerons. The more Pomerons in the event, the larger the high multiplicity tail of its spectrum. The average multiplicities of the multi-Pomeron distributions do not coincide anymore, and the local peak corresponding to the single-Pomeron exchange becomes apparent.

\subsection{Particle freeze-out}

In this section we present the distributions of coordinates and momenta for four abundant particle species, namely $\pi^{+}, K^{+}, p$ and $\Lambda+\Sigma^{0}$. Recall that the distributions are averaged over the whole ensemble of events. Therefore, the fluctuations on the event-by-event basis are disregarded. Apparently, particles can be produced in inelastic and elastic collisions, and in decays of resonances, but here we will not distinguish between the last interaction points.

The phase-space distribution of the particles on the mass shell depends on seven independent variables: coordinates $\vec{x}$, momenta $\vec{p}$, and time $t$. In both hadron-hadron interactions and central collisions of symmetric nuclei, there are only ten different coordinate pairs [26]. Two of them which give a nice visual information about the evolution of the system are shown in Figs. 10 and 11. Figure 10 displays the longitudinal position of particles versus the creation time, $\frac{d^{2} N}{d z d t}$. Calculations are done for $p p$ collisions at $\sqrt{s}=900 \mathrm{GeV}$. One can see that a large number of secondary hadrons freezes out within 

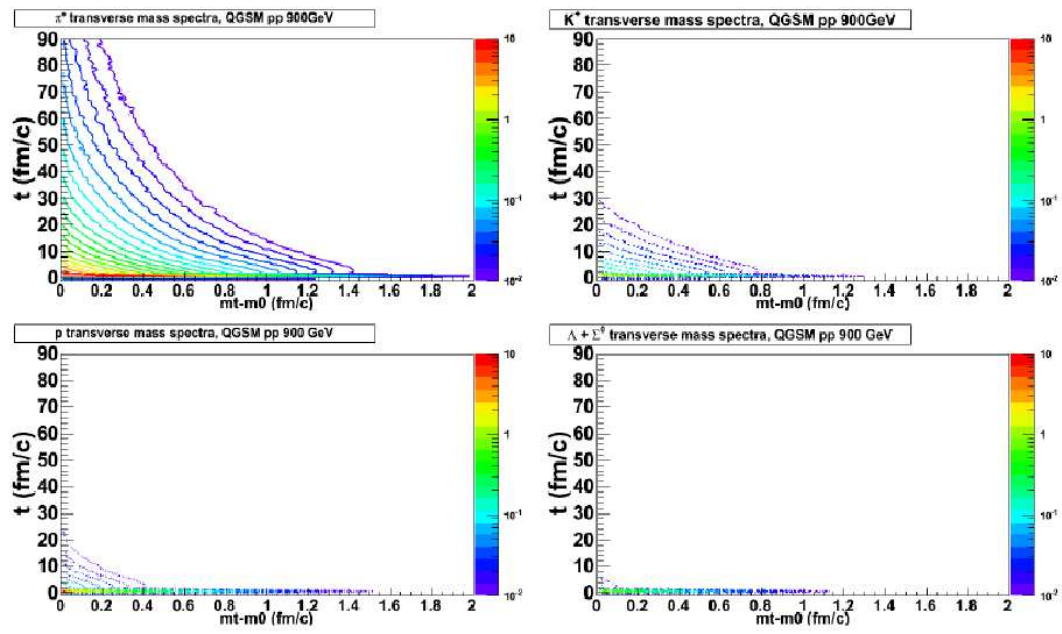

Figure 11. The same as Fig. 10 but for $d^{2} N / m_{T} d m_{T} d t$ distribution.

the first fermi-second. Particles demonstrate clear mass hierarchy: heavy particles freeze out earlier compared to the light ones, as heavy strings become less prevalent. Production of charged pions takes place up to $t \approx 18 \mathrm{fm} / c$ in the central zone of the collision, whereas for charged kaons, protons and $\Lambda$ 's these times reduce to $12 \mathrm{fm} / c, 10 \mathrm{fm} / c$, and $7 \mathrm{fm} / c$, respectively.

The transverse mass distribution, $\frac{d^{2} N}{m_{T} d m_{T} d t}$, is shown in Fig. 11. It appears that particles produced at the very beginning of the collision carry larger transverse mass and, therefore, larger transverse momentum. This finding is in line with the results of femtoscopy analysis that hadrons with the largest $p_{T}$ indicate the smallest emission zone. A second peak seen in just a few fm/c after the initial collision comes from the decays of short-lived resonances. The continuous production of hadrons from the resonance decays takes place up to quite long times. The transverse mass of the created hadrons drops almost exponentially with increasing time.

\subsection{Forward-backward multiplicity correlations}

Forward-backward multiplicity correlations between charged hadrons emitted in forward and backward hemispheres were first observed in [53]. The strength of the correlations is defined as

$$
b=\frac{\left\langle\left(n_{F}-\left\langle n_{F}\right\rangle\right)\left(n_{B}-\left\langle n_{B}\right\rangle\right)\right\rangle}{\left[\left\langle\left(n_{F}-\left\langle n_{F}\right\rangle\right)^{2}\right\rangle\left\langle\left(n_{B}-\left\langle n_{B}\right\rangle\right)^{2}\right\rangle\right]^{1 / 2}},
$$

where $n_{B}$ and $n_{F}$ represent multiplicities of charged particles in backward and forward hemispheres, respectively. Figure 12 depicts the dependence of the mean charged-particle multiplicity in the backward hemisphere $\left\langle n_{B}\right\rangle$, measured in the range $-4 \leq \eta \leq 0$, on the multiplicity in the forward hemisphere $n_{F}$ for the symmetric range $0 \leq \eta \leq 4$ at four c.m. energies, $\sqrt{s}=200 \mathrm{GeV}, 546 \mathrm{GeV}$, 


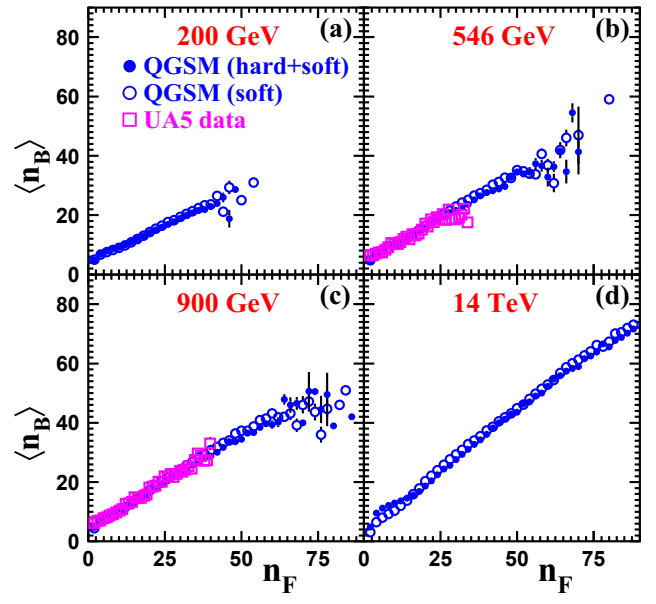

Figure 12. Backward-forward multiplicity correlations $\left\langle n_{B}\left(n_{F}\right)\right\rangle$ for $0 \leq|\eta| \leq 4$ in NSD $p p$ interactions at $\sqrt{s}=$ $200 \mathrm{GeV}$ (a), $546 \mathrm{GeV}$ (b), $900 \mathrm{GeV}$ (c) and $14 \mathrm{TeV}$ (d). Open circles denote contributions of soft processes, full symbols are for all processes. Data are from [31].

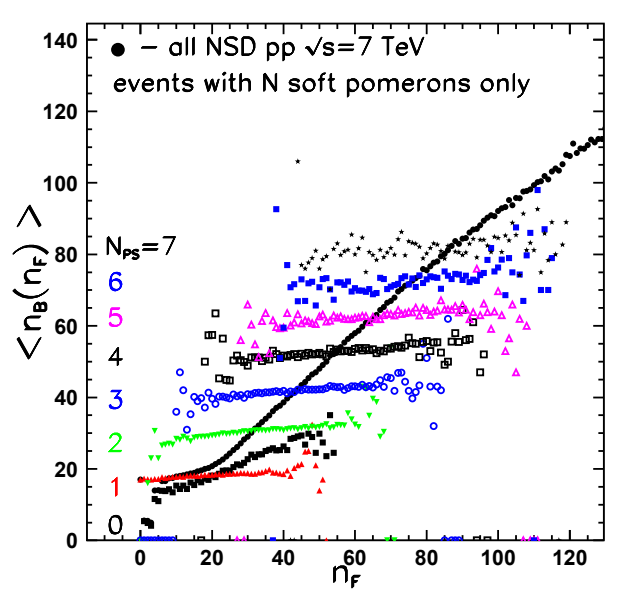

Figure 13. Backward-forward multiplicity correlations $\left\langle n_{B}\left(n_{F}\right)\right\rangle$ in QGSM calculations of NSD $p p$ interactions at $\sqrt{s}=7 \mathrm{TeV}$ proceeding via the soft Pomeron exchanges. Also shown are correlations for the collisions with fixed amount of soft Pomerons (from zero to seven).

$900 \mathrm{GeV}$, and $14 \mathrm{TeV}$. Comparison with the experimental data at $\sqrt{s}=546 \mathrm{GeV}$ and $900 \mathrm{GeV}$ shows a good agreement between the model results and the data. The dependence $\left\langle n_{B}\left(n_{F}\right)\right\rangle$ looks pretty linear at all energies,

$$
\left\langle n_{B}\left(n_{F}\right)\right\rangle=a+b n_{F},
$$

revealing a sort of long-range correlations. The correlation strength is characterized by the slope $b$, which increases with rising energy of the collisions. As shown in Fig. 12, the correlations between the $\left\langle n_{B}\right\rangle$ and $n_{F}$ are mainly determined by the soft processes.

The linear profile of the forward-backward correlations can be reproduced by the mixture of several emitting sources. Moreover, the multiplicity of the secondary hadrons should be described by the Poisson distribution, and all sources should possess different average multiplicities [31], see also $[54,55]$ and references therein. Let us sketch briefly how this picture emerges in the QGSM.

It is worth noting that the model successfully reproduces the forward-backward multiplicity correlations in $p p$ and $\bar{p} p$ collisions not only at energies above hundred $\mathrm{GeV}$, but also at energies about few $\mathrm{GeV}$ in the c.m. frame [56]. In this energy range the variety of subprocesses describing the $\bar{p} p$ interactions is more copious compared to that describing the $p p$ collisions, because of, e.g., annihilation processes, planar diagrams, and so forth. As a result, the correlation strength is stronger in $\bar{p} p$ interactions. At ultrarelativistic energies the contribution of these diagrams to the total cross section rapidly drops to zero. The difference between the $b_{c o r r}^{p p}$ and $b_{c o r r}^{\bar{p} p}$ disappears, but the slopes of the distributions become steeper at higher energies. It happens because of the increase of the variety of multi-Pomeron processes with energy. If, however, we are picking up just one particular subprocess with the fixed amount of Pomeron exchanges, the correlation slope is essentially zero, as seen in Fig. 13. In this figure the forward-backward correlations in NSD $p p$ collisions at $\sqrt{s}=7 \mathrm{TeV}$ are 
shown for the subprocesses with $N$ soft Pomerons, where $N$ varies from zero to seven. Despite the significant event-by-event multiplicity fluctuations, the slopes of the partial distributions are absent. But when we are combining the subevents with different mean multiplicities together, a nice picture of positive correlations $\left\langle n_{B}\left(n_{F}\right)\right\rangle$ arises. This circumstance explains also the nonzero slope of the distribution for events with just one soft Pomeron. Here we are mixing up several diagrams corresponding to the double diffraction displayed in Fig. 1. Detailed study of the forward-backward multiplicity correlations within the QGSM and comparison with the available experimental data can be found in [55].

\section{Conclusions}

The Monte Carlo version of the quark-gluon string model is applied for the description of protonproton collisions at energies from $\sqrt{s}=200 \mathrm{GeV}$ to $14 \mathrm{TeV}$. The model is based on Gribov's Reggeon Field Theory and employs theoretical estimates for the total cross sections, as well as for the cross sections of single diffraction and double diffraction processes. Parameters of soft and hard Pomerons are tuned to provide the best fit to the data at LHC energies. It is shown that QGSM reproduces well the bulk observables, such as yields, rapidity- and transverse momentum spectra of charged particles at all energies in question.

Several scaling hypothesis have been examined.The model indicates fulfillment of the extended longitudinal scaling accompanied by the violation of both Feynman scaling and KNO scaling. The origin of these phenomena is traced to short range momentum correlations of particles in strings and copious production of hadrons in multi-Pomeron processes. The latter leads to the formation of distinct peak in the multiplicity distribution at lower multiplicities and rise of the high-multiplicity tail of the distribution.

Although the main part of the most abundant particle species is produced at the very beginning of the collision, there is no sharp freeze-out of hadrons in $p p$ interactions. Hadron emissivity rapidly drops with time $t$. However, the production of pions in the central zone of $p p$ collisions at $\sqrt{s}=$ $900 \mathrm{GeV}$, for instance, takes place up to $t \sim 18 \mathrm{fm} / c$. For other hadron species this time is shortened, thus revealing the mass hierarchy: the heavier the particle, the faster the freeze-out proceeds. Hadrons with large transverse momentum are produced at the first $\mathrm{fm} / \mathrm{c}$, whereas soft hadrons are emitted during the whole time of evolution. Secondary peak in the $d^{2} N / m_{T} d m_{T} d t$ distribution appears because of decays of short-lived resonances.

Finally, the model was employed to study forward-backward multiplicity correlations. Note that no extra tuning of the model parameters was performed. Because of the rich set of various subprocesses at partonic level, QGSM reproduces nicely almost linear positive FB correlations in $p p$ and $\bar{p} p$ interactions in a very broad energy range. We show that for each particular subprocess the FB multiplicity correlations are absent despite of the significant multiplicity fluctuations on event-by-event basis. But when we add up all these events with different average multiplicities, the picture of strong positive FB correlations emerges.

\section{Acknowledgements}

We would like to thank B. Kopeliovich, V. Khose, E. Levin, L. Lipatov, J. Nyiri, and V. Fadin for the fruitful discussions and valuable comments. J.B. thanks the German Research Foundation (DFG) for the financial support through the Project BL 1286/2-1. L.B. acknowledges financial support of the Alexander von Humboldt Foundation. 


\section{References}

[1] A.B. Kaidalov, Phys. Lett. B 116, 459 (1982);

A.B. Kaidalov and K.A. Ter-Martirosyan, Phys. Lett. B 117, 247 (1982).

[2] A. Capella, U. Sukhatme, C.-I. Tan, and J. Tran Thanh Van, Phys. Rep. 236, 225 (1994).

[3] V.N. Gribov, Sov. Phys. JETP 26, 414 (1968).

[4] G. t'Hooft, Nucl. Phys. B 72, 461 (1974).

[5] G. Veneziano, Phys. Lett. B 52, 220 (1974).

[6] N.S. Amelin and L.V. Bravina, Sov. J. Nucl. Phys. 51, 133 (1990).

[7] V. Abramovskii, V. Gribov, O. Kancheli, Sov. J. Nucl. Phys. 18, 308 (1974).

[8] A. B. Kaidalov, Surveys in High Energy Phys. 13, 265 (1999).

[9] M. Baker, K. A. Ter-Martirosyan, Phys. Rep. 28C, 1 (1976).

[10] O.V. Kancheli, JETP Lett. 11, 267 (1970);

A.H. Mueller, Phys. Rev. D 2, 2963 (1970).

[11] Y. V. Kovchegov and K. Tuchin, Phys. Rev. D 65, 074026 (2002);

E. Levin and A. Prygarin, Phys. Rev. C 78, 065202 (2008).

[12] K. Werner, F.-M. Liu, and T. Pierog, Phys. Rev. C 74, 044902 (2006).

[13] R. Engel, J. Ranft, and S. Roesler, Phys. Rev. D 52, 1459 (1995).

[14] S. Ostapchenko, Nucl. Phys., Proc. Suppl. 151, 143 (2006); Phys. Rev. D 83, 014018 (2011).

[15] A.B. Kaidalov and M.G. Poghosyan, Eur. Phys. J. C 67, 397 (2010).

[16] J. Bleibel, L.iV. Bravina, and E.E. Zabrodin, Phys. Rev. D 93, 114012 (2016).

[17] E. Gotsman, E. Levin, U. Maor, and J. S. Miller, Eur. Phys. J. C 57, 689 (2008).

[18] E. Gotsman, E. Levin, and U. Maor, Eur. Phys. J. C 71, 1553 (2011).

[19] M. G. Ryskin, A. D. Martin, and V. A. Khoze, Eur. Phys. J. C 54, 199 (2008).

[20] M. G. Ryskin, A. D. Martin, V. A. Khoze, and A. G. Shuvaev, J. Phys. G 36, 093001 (2009).

[21] R. D. Field, R. P. Feynman, Nucl. Phys. B 136, 1 (1978).

[22] N.S. Amelin, E.F. Staubo, and L.P. Csernai, Phys. Rev. D 46, 4873 (1992).

[23] N.S. Amelin et al., Phys. Rev. C 47, 2299 (1993).

[24] L. Bravina, L.P. Csernai, P. Levai, D. Strottman, Phys. Rev. C 50, 2161 (1994).

[25] L.V. Bravina, Phys. Lett. B 344, 49 (1995).

[26] L.V. Bravina et al., Phys. Rev. C 60, 044905 (1999).

[27] E.E. Zabrodin et al., Phys. Lett. B 508, 184 (2001).

[28] E.E. Zabrodin et al., Phys. Rev. C 63, 034902 (2001).

[29] L.V. Bravina et al., Phys. Rev. C 78, 014907 (2008).

[30] G. Burau et al., Phys. Rev. C 71, 054905 (2005).

[31] G. J. Alner et al. (UA5 Collab.), Phys. Rep. 154, 247 (1987).

[32] G. Arnison et al. (UA1 Collab.), Phys. Lett. B 118, 167 (1982);

C. Albajar et al. (UA1 Collab.), Nucl. Phys. B 335, 261 (1990).

[33] F. Abe et al. (CDF Collab.), Phys. Rev. Lett. 61, 1819 (1988); Phys. Rev. D 41, R2330 (1990).

[34] T. Alexopoulos et al. (E735 Collab.), Phys. Rev. D 48, 984 (1993).

[35] ALICE Collaboration (K. Aamodt et al.), Eur. Phys. J. C 68, 89 (2010).

[36] ALICE Collaboration (K. Aamodt et al.), Eur. Phys. J. C 68, 345 (2010).

[37] ALICE Collaboration (K. Aamodt et al.), Phys. Lett. B 693, 53 (2010).

[38] ALICE Collaboration (K. Aamodt et al.), Report No. ALICE-PUBLIC-2013-001.

[39] ALICE Collaboration (J. Adam et al.), Phys. Lett. B 753, 319 (2016). 
[40] CMS Collaboration (K. Khachatryan et al.), J. High Energy Phys. 02, 041 (2010).

[41] CMS Collaboration (K. Khachatryan et al.), Phys. Rev. Lett. 105, 022002 (2010).

[42] CMS Collaboration (K. Khachatryan et al.), Phys. Lett. B 751, 143 (2015).

[43] CMS and TOTEM Collaborations (S. Chatrchyan et al.), Eur. Phys. J. C 74, 3053 (2014).

[44] L. McLerran and M. Praszalowicz, Acta Phys. Polon. B 41, 1917 (2010).

[45] E. Levin and A.H. Rezaeian, Phys. Rev. D 82, 014022 (2010).

[46] R. Feynman, Phys. Rev. Lett. 23, 1415 (1969);

R. Feynman, Photon-hadron interactions (Benjamin, NY, 1972).

[47] W. Busza, J. Phys. G 35, 044040 (2008).

[48] G.J. Alner et al. (UA5 Collab.), Z. Phys. C 33, 1 (1986).

[49] J. Cleymans, J. Strümpfer, and L. Turko, Phys. Rev. C 78, 017901 (2008).

[50] Z. Koba, H.B. Nielsen, and P. Olesen, Nucl. Phys. B 40, 317 (1972).

[51] E.M. Levin and M.G. Ryskin, Yad. Fiz. 19, 389 (1974).

[52] V. Zaccolo et al. (ALICE Collab.), Nucl. Phys. A 956, 529 (2016).

[53] C.J. Alner et al. (UA5 Collab.), Nucl. Phys. B 291, 445 (1987).

[54] V.V. Vechernin, these Proceedings.

[55] L. Bravina, J. Bleibel, R. Emaus, E. Zabrodin, these Proceedings.

[56] L.V. Bravina et al., Sov. J. Nucl. Phys. 50, 245 (1989). 